\title{
Corneal biomechanical properties in healthy children measured by corneal visualization scheimpflug technology
}

\author{
Miao He ${ }^{1 \dagger}$, Hui Ding ${ }^{2 \dagger}$, Hong He ${ }^{2}$, Chi Zhang ${ }^{1}$, Liangping Liu ${ }^{1}$ and Xingwu Zhong ${ }^{1,2^{*}}$ (D)
}

\begin{abstract}
Background: The aim of this study was to evaluate corneal biomechanical properties in a population of healthy children in China using corneal visualization Scheimpflug technology (CST).

Methods: All children underwent complete bi-ocular examinations. CST provided intraocular pressure (IOP) and corneal biomechanical parameters, including time, velocity, length and deformation amplitude at first applanation (A1T, A1V, A1L, A1DA), at second applanation (A2T, A2V, A2L, A2DA), highest concavity time (HCT), maximum deformation amplitude (MDA), peak distance (PD), and radius of curvature (RoC). Pearson correlation analysis was used to assess the impacts of demographic factors, central corneal thickness (CCT), spherical equivalent (SE), and IOP on corneal biomechanics.

Results: One hundred eight subjects (32 girls and 76 boys) with the mean age of $10.80 \pm 4.13$ years (range 4 to 18 years) were included in the final analyses. The right and left eyes were highly symmetrical in SE ( $p=0.082)$, IOP $(p=0.235)$, or CCT ( $p=0.210$ ). Mean A1T of the right eyes was $7.424 \pm 0.340 \mathrm{~ms}$; the left eyes $7.451 \pm 0.365 \mathrm{~ms}$. MDA was $0.993 \pm 0.102 \mathrm{~mm}$ in the right eyes and $0.982 \pm 0.100 \mathrm{~mm}$ in the left eyes. Mean HCT of the right eyes was $16.675 \pm 0.502 \mathrm{~ms}$; the left eyes $16.735 \pm 0.555 \mathrm{~ms}$. All CST parameters of both eye were remarkably symmetrical with the exception of A2L ( $p=0.006)$, A1DA $(p=0.025)$. The majority of CST parameters of both eyes were significantly correlated with CCT and IOP $(p<0.05)$. However, age, SE, and sex exert little influence on the CST measurements.
\end{abstract}

Conclusions: This study found interocular symmetry in corneal biomechanics in healthy children eyes. Several CST biomechanical parameters in children are modified by CCT and IOP.

Keywords: Corvis ST, Corneal biomechanics, Corneal deformation, Symmetry, Chinese, Children

\section{Background}

Corneal biomechanical properties play an important role in maintaining the shape and transparency of the cornea [1]. Various factors influence the corneal biomechanics such as hydration, elasticity, viscosity, and the thickness of corneal stroma [2]. Among the five layers of cornea, the stroma was considered to be the primary loadcarrying layer. A large number of studies demonstrated that the corneal biomechanics altered significantly in eyes with keratoconus, Fuchs corneal dystrophy, glaucoma, post refractive surgery $[3,4]$. Increase

\footnotetext{
* Correspondence: zhongxwu@mail.sysu.edu.cn

${ }^{\dagger}$ Equal contributors

'Zhongshan Ophthalmic Center and State Key Laboratory of Ophthalmology, Sun Yat-sen University, 54S Xianlie Road, 510060 Guangzhou, China

${ }^{2}$ Hainan Eye Hospital, Zhongshan Ophthalmic Center, Sun Yat-sen University, Haikou, China
}

knowledge of corneal biomechanics is essential for the of the risk of ectasia after refractive surgery.

Two commercial devices are available for characterizing the biomechanical properties of the cornea in vivo currently, i.e. the Ocular Response Analyzer (ORA, Reichert, Buffalo, New York, USA) and corneal visualization Scheimpflug technology (Corvis ST [CST], Oculus, Wetzlar, Germany). The ORA was introduced in 2004 based on bidirectional applanation tonometry, which rovides intraocular pressure (IOP) and two corneal biomechanical parameters: corneal hysteresis $(\mathrm{CH})$ and corneal resistance factor (CRF) [5-7]. However, the ORA was criticized for poor repeatability and the fact that it is easily affected by corneal morphological parameters $[8,9]$. In contrast to the ORA, the newer CST was based on 
corneal deformation measurement using Scheimpflug imaging technology, which records the whole process of cornea deformation following an air puff and provides over ten biomechanical parameters.

Children are a special population whose physiology and pathology are greatly different with adults. Knowledge of corneal biomechanics in children can help to master the ocular geometry and refractive status of the child. In the previous study of corneal biomechanics achieved by ORA, researchers have found that lower $\mathrm{CH}$ to be significantly associated with longer $\mathrm{AL}$, poor correlation has been reported in adult studies [10]. Besides, ORA parameters could both reflect anterior geometry such as iris concavity $[6,7]$ and posterior geometry, research have confirmed that in myopic children the average RNFL thickness and rim area correlated positively with $\mathrm{CH}$ whereas the average cup-to-disc area ratio correlated negatively with $\mathrm{CH}$ [11]. Considering the reliability and reproducibility of ORA parameter were poor compared with CST, thus it is necessary to investigate the relationship between CST parameter and corneal biomechanics in children. The symmetry of the paired organs has been used as a tool to evaluate certain disease. Understanding the normal range of differences between eyes will help inform analysis of what degree of asymmetry between eyes can be considered possibly pathologic. Although several studies have investigated the corneal biomechanics in adults [3, 4, 12-18], we did not found any reports on corneal biomechanical properties in children using CST and the symmetry of biomechanics parameters. To fill this gap, we performed a cross-sectional study to assess the corneal biomechanics in healthy Chinese children using CST and evaluate the inter-ocular symmetry and associations of CST parameters with other factors.

\section{Methods \\ Subjects}

This observational and cross-sectional study was completed at the Hainan Eye Hospital of the Zhongshan Ophthalmic Center in China from July to August, 2015. The study followed the tenets of the Declaration of Helsinki and the protocol was reviewed and approved by the Ethics Review Board of Hainan Eye Hospital of the Zhongshan Ophthalmic Center (HNEH2015-015). Informed consents were obtained from the subjects' parents before examination.

Healthy children without ocular disease (except refractive errors) were recruited at the hospital setting. The children were composed of 1) children came to hospital for regular optometry screening; 2) hospital employees' children; 3) children whose parents visiting the hospital. Subjects with any systemic and/or ocular diseases that influence corneal evaluation, such as diabetes, connective tissue disorders, dry eye, uveitis, corneal ulcers, glaucoma, cataract, or retinopathy were excluded from the study. The children who wore contact lenses were also excluded. The ocular examinations were performed under the assistance of their parents.

\section{Ocular and CST examinations}

All children underwent complete bi-ocular examinations, including slit-lamp evaluation, auto refractometer (RM-8900, TOPCON, Japan), and CST imaging (Oculus Optikgeräte $\mathrm{GmbH}$, Wetzlar, Germany). Demographic data were recorded such as date of birth, sex, history of disease.

All CST examinations were conducted by an experienced technician, who was blind to the study protocol. The right eye was firstly examined, then the left eye underwent CST scan. Children were asked to adjust their chin and forehead to an appropriate height, then focus on the red light emitting diode (LED). The exam is programmed for automatic release when alignment is achieved with the first Purkinje reflex of the cornea. The CST takes more than 4,300 frames of the central 8-mm horizontal portion per second with a high-speed Scheimpflug camera, which records and displays the entire response of cornea deformation to an air puff from the instrument. The air puff is $25 \mathrm{Kpa}$ and lasts $20 \mathrm{~ms}$. Finally, 140 digital frames are obtained. Each image has 576 possible measuring points. The cornea experiences four distinct statuses, i.e. first applanation (A1), the highest concavity (HC), second applanation (A2) and natural status (Fig. 1). The latest analyzing software (Corvis_ST_1.2r1126) was used in this study. Only the images with an "OK" in Quality Specification (QS) by built-in software were included in the final analysis.

\section{Statistical analysis}

Statistical analysis was performed using SPSS 20.0 (SPSS, Inc., Chicago, IL). The Kolmogorov-Smirnov test was used to verify the distribution of normality. All results of continuous variables were presented as mean \pm standard deviation (SD). The paired $t$ test and intraclass correlation coefficient (ICC) were used to compare the measurements of the right and left eye. A Pearson correlation analysis was used to evaluate the relationships between age, gender, IOP, CCT, SE, and CST parameters. The level of significance was set at $5 \%$ with two sides.

\section{Results}

Initially, a total of 140 children were invited for this study, but 20 refused to take part in this study. Among the 240 eyes (120 children), 40 eyes were excluded because inadequate quality of the CST images. Thus, 200 eyes of 108 children with 32 girls and 76 boys were included in the final analyses, with the mean age of 


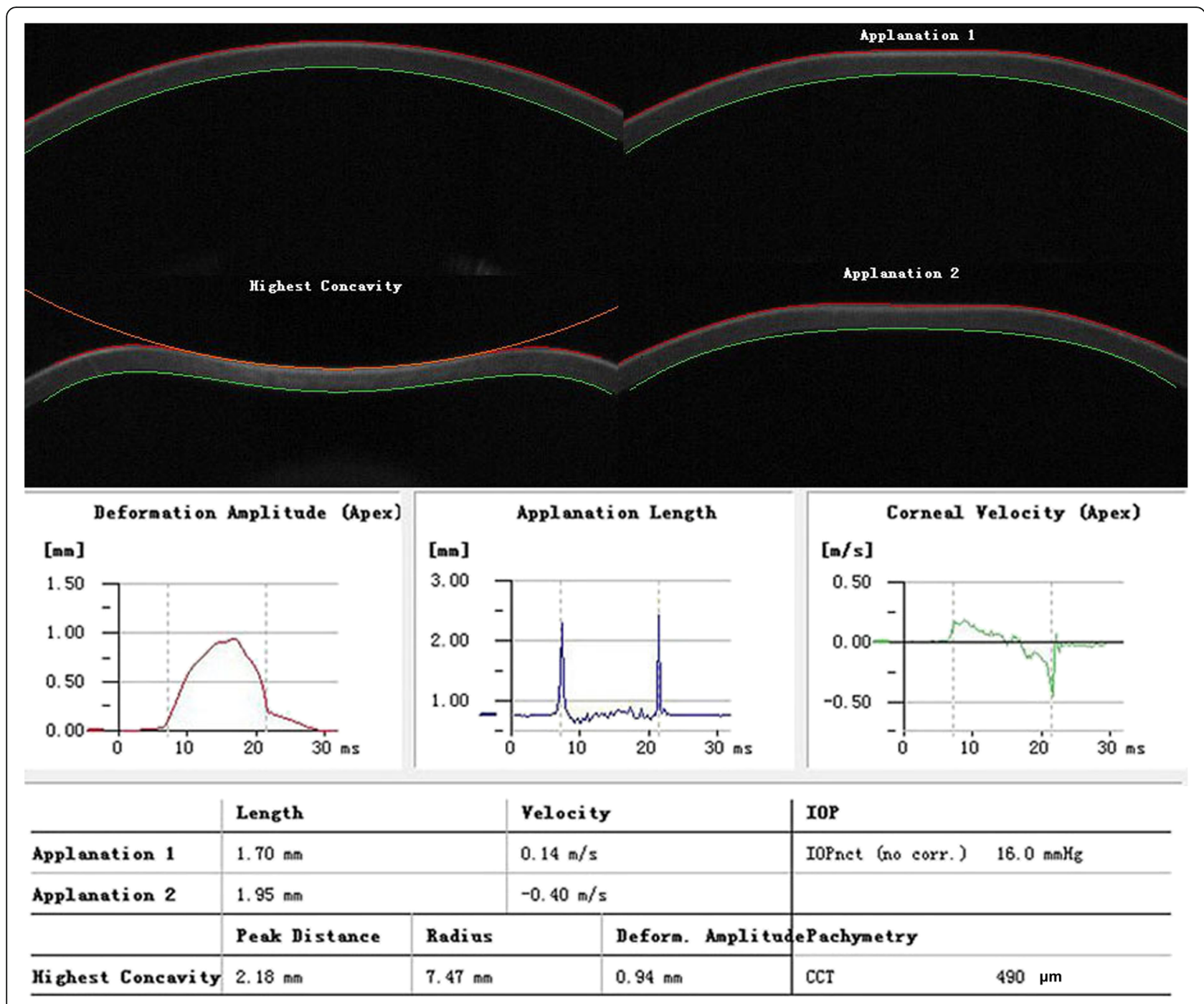

Fig. 1 Diagram of corneal response parameters on the images from the corneal visualization Scheimpflug technology

$10.80 \pm 4.13$ years (range 4 to18 years). Table 1 summarizes the demographic and clinical data of the subjects. The right and left eyes were highly symmetrical in SE $(p=0.082)$, IOP $(p=0.235)$, or CCT $(p=0.210)$ (Table 1$)$.

The mean values of CST parameters are presented in Table 2. Mean A1T of the right eyes was $7.424 \pm 0.340 \mathrm{~ms}$; the left eyes $7.451 \pm 0.365 \mathrm{~ms}$. MDA was $0.993 \pm 0.102 \mathrm{~mm}$ in the right eyes and $0.982 \pm 0.100 \mathrm{~mm}$ in the left eyes. Mean HCT of the right eyes was $16.675 \pm 0.502 \mathrm{~ms}$; the left eyes16.735 $\pm 0.555 \mathrm{~ms}$. All CST parameters of both eye were similar with the exception of A2L $(p=0.006)$, A1DA $(p=0.025)$. These results revealed that the right eyes and left eyes were highly symmetric in most CST parameters.

Table 3 shows the results of correlation analyses. Age and gender were not significantly associated with most CST parameters in the right eyes or left eyes. CCT was significantly and positively correlated to A1T, A1DA of the both eyes. SE was positively correlated to HCT,
Table 1 Demographic and ocular characteristics of subjects

\begin{tabular}{lll}
\hline Variables & Mean \pm SD & $P^{*}$ \\
\hline Age (years) & $10.80 \pm 4.13$ & 0.082 \\
SE (diopters) & & \\
$\quad$ Right eye & $-3.13 \pm 3.69$ & \\
$\quad$ Left eye & $-2.82 \pm 3.77$ & 0.235 \\
IOP (mmHg) & & \\
$\quad$ Right eye & $15.83 \pm 2.84$ & 0.210 \\
Left eye & $16.03 \pm 3.03$ & \\
CCT ( $\mu$ m) & & \\
$\quad$ Right eye & $549.62 \pm 30.96$ & \\
Left eye & $550.83 \pm 32.49$ & \\
\hline
\end{tabular}

SE spherical equivalent, IOP intraocular pressure, $C C T$ central corneal thickness, $P P$ values, *paired $t$ test 
Table 2 Mean values and inter-eye symmetry of corneal biomechanical parameters in subjects

\begin{tabular}{|c|c|c|c|c|c|}
\hline Parameters & Right eye & Left eye & Mean difference $(95 \% \mathrm{Cl})$ & $P^{*}$ & ICC \\
\hline $\mathrm{A} 1 \mathrm{~T}, \mathrm{~ms}$ & $7.424 \pm 0.340$ & $7.451 \pm 0.365$ & $-0.028(-0.067$ to 0.012$)$ & 0.167 & 0.906 \\
\hline $\mathrm{A} 1 \mathrm{~L}, \mathrm{~mm}$ & $1.729 \pm 0.066$ & $1.726 \pm 0.094$ & $0.003(-0.016$ to 0.021$)$ & 0.784 & 0.415 \\
\hline $\mathrm{A} 1 \mathrm{~V}, \mathrm{~m} / \mathrm{s}$ & $0.139 \pm 0.020$ & $0.138 \pm 0.026$ & 0.001 (-0.004 to 0.005$)$ & 0.861 & 0.592 \\
\hline A1DA, mm & $0.119 \pm 0.009$ & $0.116 \pm 0.011$ & 0.002 (0.001 to 0.004$)$ & 0.025 & 0.685 \\
\hline $\mathrm{A} 2 \mathrm{~T}, \mathrm{~ms}$ & $21.586 \pm 0.869$ & $21.401 \pm 1.216$ & $0.184(-0.097$ to 0.466$)$ & 0.197 & 0.224 \\
\hline $\mathrm{A} 2 \mathrm{~L}, \mathrm{~mm}$ & $1.688 \pm 0.319$ & $1.554 \pm 0.383$ & 0.133 (0.038 to 0.228 ) & 0.006 & 0.199 \\
\hline $\mathrm{A} 2 \mathrm{~V}, \mathrm{~m} / \mathrm{s}$ & $-0.334 \pm 0.102$ & $-0.334 \pm 0.078$ & $0.0002(-0.022$ to 0.022$)$ & 0.985 & 0.451 \\
\hline A2DA, mm & $0.367 \pm 0.131$ & $0.372 \pm 0.141$ & $-0.004(-0.038$ to 0.030$)$ & 0.800 & 0.396 \\
\hline $\mathrm{HCT}$, ms & $16.675 \pm 0.502$ & $16.735 \pm 0.555$ & $-0.060(-0.197$ to 0.077$)$ & 0.389 & 0.137 \\
\hline $\mathrm{PD}, \mathrm{mm}$ & $2.940 \pm 1.083$ & $2.686 \pm 1.080$ & 0.253 (-0.037 to 0.544$)$ & 0.086 & 0.027 \\
\hline $\mathrm{RoC}, \mathrm{mm}$ & $6.470 \pm 1.295$ & $6.438 \pm 1.318$ & $0.032(-0.265$ to 0.330$)$ & 0.831 & 0.449 \\
\hline MDA, mm & $0.993 \pm 0.102$ & $0.982 \pm 0.100$ & 0.011 (-0.002 to 0.024$)$ & 0.088 & 0.872 \\
\hline
\end{tabular}

Data were presented as mean \pm standard deviation

95\% Cl 95\% confidential interval, *paired $t$ test, ICC intraclass correlation coefficient

$A 1 T$ Time from start to the first flatterning of cornea, $A 1 L$ Length of flattened cornea in the first applanation, A1V Inward velocity of the cornea in the first applanation, A1DA Cornea apex displacement in vertical direction at first applanation, $A 2 T$ Time from start to second flattening of the cornea, A2L Length of flattened cornea in the second applanation, A2V Outward velocity of the cornea in the second applanation, A2DA Cornea apex displacement in vertical direction at second applanation, HCT Time from start to the highest concavity of cornea, PD Distance between the corneal peaks at maximal concavity, RoC Radius of curvature of the corneal at highest concavity, MDA Cornea apex displacement in vertical direction at highest concavity

Bold indicates statistical significance $(P<0.05)$

A2DA of the right eye and MDA, PD, A2DA of the left eye. IOP was positively associated with A1T, A1L, A2V, RoC, and A1DA in both eyes, while negatively related to MDA, A1V, A2T of both eyes.

\section{Discussion}

We evaluated the corneal biomechanical properties in a population of healthy children in China. As far as we know, this is the first report of quantitative assessment of the corneal biomechanics in children population using CST. We used the newly updated CST software, which provided two more parameters (A1DA and A2DA) than previous versions. This helped to measure corneal deformation more comprehensively. We also assessed the symmetry of corneal biomechanics between the both eyes and found obviously interocular symmetry in SE, CCT, IOP, and corneal biomechanics in healthy children eyes. We also found that several CST biomechanical parameters in children are modified by CCT and IOP, while age, SE, and sex exert little influence on the CST measurements in this population.

Our observations of interocular symmetric biometry were consistent with previous studies [19-21]. Using ORA, Zheng et al. [22] demonstrated an obvious symmetry of $\mathrm{CH}$ and CRF in bilateral rabbit corneas. We also identified two asymmetrical parameters in the sample, i.e. A2L and A1DA. The corneal stroma is composed of highly arranged collagen fibrils [23]. Wideangle $x$-ray scattering study demonstrated that distribution of the aligned fibrils in the cornea generally was symmetrical in the both eyes, but the mass of preferentially aligned collagen in the corneal stroma appears to be distributed differently between left and right eyes [24]. Thus, we postulate that the asymmetry of A2L and A1DA may be affected by asymmetry arrangement of corneal collagen. Besides the poor repeatability and reproducibility of A2L and A1DA which were reported by previous studies [25-27] also could explain some of differences found between the right eye and the left eye. However, further studies are warrant to clarify the exactly mechanism.

One interesting finding is that age was not correlated with CST parameters in children. Previous studies have investigated children's corneal biomechanics using ORA, but Huang et al. [10], Shah et al. [6, 7] and Buenoet al. [11] obtained controversial results. In Brazil adults, Valbon et al. [14] reported that age was positively correlated to $\mathrm{HCT}(P=0.048)$. In Chinese adults, Wang and his colleagues also revealed a positively correlation between age and A2L $(p=0.03)$ [18]. An increase in crosslinking fibers in corneal stroma with age was observed using X-ray, suggesting a stiffer cornea in older people $[28,29]$ The inconsistency between the present study and studies on adults using CST indicating the influence of age on CST parameters depended on age levels.

We found the most influential factor of CST parameters was IOP. We showed a positive correlation between IOP and A1T, A1L, A2V, RoC, and A1DA, while it indicated a negative correlation between IOP and MDA, A1V, A2T of both eyes, and PD of left eyes. These results suggest that corneal deformability decreased with an increasing IOP. Previous ORA studies 
Table 3 Correlations between corneal biomechanical parameters and demographic or ocular parameters

\begin{tabular}{|c|c|c|c|c|c|c|c|c|c|c|}
\hline & \multicolumn{2}{|l|}{ Age } & \multicolumn{2}{|l|}{$\mathrm{CCT}$} & \multicolumn{2}{|l|}{ Sex } & \multicolumn{2}{|l|}{$\underline{\text { SE }}$} & \multicolumn{2}{|l|}{ IOP } \\
\hline & $r$ & P & $r$ & $P$ & $r$ & $P$ & $r$ & $P$ & $r$ & $P$ \\
\hline \multicolumn{11}{|l|}{ Right eye } \\
\hline $\mathrm{A} 1 \mathrm{~T}, \mathrm{~ms}$ & 0.071 & 0.464 & 0.272 & 0.004 & 0.145 & 0.131 & -0.104 & 0.278 & 0.995 & $<0.001$ \\
\hline $\mathrm{A} 1 \mathrm{~L}, \mathrm{~mm}$ & 0.102 & 0.288 & 0.281 & 0.003 & 0.027 & 0.783 & 1.000 & 0.298 & 0.170 & 0.076 \\
\hline $\mathrm{A} 1 \mathrm{~V}, \mathrm{~m} / \mathrm{s}$ & -0.035 & 0.719 & -0.170 & 0.075 & -0.043 & 0.655 & 0.047 & 0.626 & -0.735 & $<0.001$ \\
\hline $\mathrm{A} 1 \mathrm{DA}, \mathrm{mm}$ & -0.137 & 0.152 & 0.407 & $<0.001$ & -0.075 & 0.437 & 0.167 & 0.079 & 0.509 & $<0.001$ \\
\hline $\mathrm{A} 2 \mathrm{~T}, \mathrm{~ms}$ & 0.014 & 0.888 & -0.253 & 0.010 & -0.205 & 0.038 & 0.125 & 0.205 & -0.355 & $<0.001$ \\
\hline $\mathrm{A} 2 \mathrm{~L}, \mathrm{~mm}$ & 0.018 & 0.857 & 0.089 & 0.371 & -0.103 & 0.298 & -0.065 & 0.511 & 0.148 & 0.134 \\
\hline $\mathrm{A} 2 \mathrm{~V}, \mathrm{~m} / \mathrm{s}$ & 0.027 & 0.788 & 0.114 & 0.252 & -0.034 & 0.736 & 0.115 & 0.242 & 0.525 & $<0.001$ \\
\hline $\mathrm{A} 2 \mathrm{DA}, \mathrm{mm}$ & -0.117 & 0.239 & 0.311 & 0.001 & 0.116 & 0.242 & 0.409 & $<0.001$ & -0.158 & 0.112 \\
\hline $\mathrm{HCT}, \mathrm{ms}$ & 0.014 & 0.882 & 0.063 & 0.513 & 0.076 & 0.429 & 0.234 & 0.014 & -0.122 & 0.205 \\
\hline $\mathrm{PD}, \mathrm{mm}$ & -0.282 & 0.003 & -0.003 & 0.974 & -0.131 & 0.173 & -0.049 & 0.608 & -0.149 & 0.120 \\
\hline $\mathrm{RoC}, \mathrm{mm}$ & 0.136 & 0.155 & 0.125 & 0.194 & -0.021 & 0.824 & 0.070 & 0.468 & 0.319 & 0.001 \\
\hline MDA, mm & 0.026 & 0.789 & -0.139 & 0.147 & -0.113 & 0.241 & -0.071 & 0.456 & -0.801 & $<0.001$ \\
\hline \multicolumn{11}{|l|}{ Left eye } \\
\hline $\mathrm{A} 1 \mathrm{~T}, \mathrm{~ms}$ & -0.038 & 0.696 & 0.333 & $<0.001$ & 0.074 & 0.445 & -0.041 & 0.668 & 0.995 & $<0.001$ \\
\hline VA1L, mm & -0.078 & 0.425 & 0.104 & 0.286 & -0.034 & 0.729 & 0.175 & 0.067 & 0.197 & 0.041 \\
\hline $\mathrm{A} 1 \mathrm{~V}, \mathrm{~m} / \mathrm{s}$ & -0.077 & 0.426 & 0.033 & 0.735 & -0.052 & 0.595 & 0.039 & 0.690 & -0.444 & $<0.001$ \\
\hline $\mathrm{A} 1 \mathrm{DA}, \mathrm{mm}$ & -0.083 & 0.395 & 0.426 & $<0.001$ & 0.031 & 0.749 & 0.076 & 0.428 & 0.538 & $<0.001$ \\
\hline $\mathrm{A} 2 \mathrm{~T}, \mathrm{~ms}$ & -0.005 & 0.957 & -0.041 & 0.684 & 0.033 & 0.740 & 0.077 & 0.434 & -0.383 & $<0.001$ \\
\hline $\mathrm{A} 2 \mathrm{~L}, \mathrm{~mm}$ & 0.153 & 0.123 & 0.249 & 0.011 & -0.020 & 0.838 & 0.064 & 0.516 & 0.158 & 0.112 \\
\hline $\mathrm{A} 2 \mathrm{~V}, \mathrm{~m} / \mathrm{s}$ & -0.026 & 0.801 & 0.193 & 0.055 & 0.154 & 0.126 & 0.100 & 0.315 & 0.458 & $<0.001$ \\
\hline $\mathrm{A} 2 \mathrm{DA}, \mathrm{mm}$ & -0.091 & 0.370 & 0.026 & 0.798 & 0.093 & 0.355 & 0.233 & 0.019 & -0.152 & 0.130 \\
\hline $\mathrm{HCT}, \mathrm{ms}$ & -0.101 & 0.298 & 0.051 & 0.601 & 0.129 & 0.183 & 0.084 & 0.381 & -0.119 & 0.218 \\
\hline $\mathrm{PD}, \mathrm{mm}$ & 0.074 & 0.448 & -0.159 & 0.101 & -0.122 & 0.208 & -0.242 & 0.011 & -0.193 & 0.046 \\
\hline $\mathrm{RoC}, \mathrm{mm}$ & 0.059 & 0.545 & 0.161 & 0.096 & 0.008 & 0.937 & 0.249 & 0.090 & 0.260 & 0.007 \\
\hline $\mathrm{MDA}, \mathrm{mm}$ & 0.078 & 0.421 & -0.167 & 0.084 & -0.042 & 0.667 & -0.257 & 0.007 & -0.798 & $<0.001$ \\
\hline
\end{tabular}

IOP Intraocular pressure, CCT Central corneal thickness, SE spherical equivalent

$A 1 T$ Time from start to the first flatterning of cornea, $A 1 L$ Length of flattened cornea in the first applanation, $A 1 V$ Inward velocity of the cornea in the first applanation, A1DA Cornea apex displacement in vertical direction at first applanation, $A 2 T$ Time from start to second flattening of the cornea, $A 2 L$ Length of flattened cornea in the second applanation, $A 2 \mathrm{~V}$ Outward velocity of the cornea in the second applanation, $A 2 D A$ Cornea apex displacement in vertical direction at second applanation, HCT Time from start to the highest concavity of cornea, PD Distance between the corneal peaks at maximal concavity, RoC Radius of curvature of the corneal at highest concavity, MDA Cornea apex displacement in vertical direction at highest concavity

Bold indicates statistical significance $(P<0.05)$

have shown that IOP has important influences on $\mathrm{CH}$ and CRF [5-7]. The recent studies using CST also found glaucoma patients with high IOP have less deformable corneas $[4,16,18]$. We demonstrated that CCT was significantly and positively correlated to A1T, A1DA of both eyes, which was generally consistent with the deformation properties in adults $[3,4,13]$.

The relationship between SE and CST parameters was complex. In the right eye, SE has a positive correlation with HCT and A2DA. In the left eye, SE has a negative relationship with MDA and $\mathrm{PD}$, whereas has a positive correlation with A2DA. In the Italian adults, Lanza et al. [12] reported that SE was not correlated to A1 and A2 parameters and MDA in the left eye. The discrepancy may be related to different ethnicity, version of CST software, or age distribution of subjects. It was reported that corneal deformation behavior were affected by composition of collagen fibrils, hydration, and other as-yetunknown factors [30]. More studies with large sample size are warranted to clarify the correlation of SE with CST parameters.

Limitations should not be ignored in the present study. First, this is a cross-sectional study with relative small sample size, thus our results need to be further confirmed by longitudinal cohort studies with larger sample size. Second, diurnal variation may exist because $\mathrm{CH}$ and CRF of ORA showed significant diurnal variation [31]. Third, axial length (AL) was not measured. 
Longer AL was reported to be significantly associated with lower $\mathrm{CH}(p<0.001)$ [10]. The association between CST parameters and AL should be assessed in future because AL changes with the aging of children and is closely related to the progress of myopia. Besides, asymmetry found between the right eye and left eyes could not be confirmed since it was possible that not the real asymmetry existed but the poor reliability and reproducibility of some CST parameters cause the difference. Fourth, the sample subjects were not population based. For a mean difference of A1DA of 0.002, it was estimated that 215 subjects were required based on power of $90 \%$ and alpha of $5 \%$, with a precision (standard deviations of both eyes) of 0.009. Our 108 subjects was fewer than estimated, however, the difference in A1DA between eyes was statistically significant so that the small sample size instead strengthen our findings. Thus, even inclusion of larger subjects in future study, the same conclusions will be expected. Finally, whether correlations between CST biomechanical parameters and CST derived IOP/CCT may in part be driven by the same instrument deriving these measures remain unclear. Thus, the exact implications of CST parameters needed to be elucidated.

\section{Conclusions}

The present study evaluated the corneal biomechanical properties in healthy children in China using CST. The corneal biomechanical properties were generally symmetric in bilateral eyes. CCT and IOP have shown to have important influence on CST biomechanical parameters, while age, SE, and sex exert little influence on the CST measurements in children. Further studies with large sample size are needed to assess the corneal biomechanics in children population.

\section{Abbreviations \\ A1: First applanation; A1DA: Deformation amplitude at first applanation; A1L: Length at first applanation; A1T: Time at first applanation; A1V: Velocity at first applanation; A2: Second applanation; A2DA: Deformation amplitude at second applanation; A2L: Length at second applanation; A2T: Time at second applanation; A2V: Velocity at second applanation; AL: Axial length; CCT: Central corneal thickness; $\mathrm{CH}$ : Corneal hysteresis; CRF: Corneal resistance factor; HC: The highest concavity; HCT: Highest concavity time; IOP: Intraocular pressure; LED: Light emitting diode; MDA: Maximum deformation amplitude; PD: Peak distance; RoC: Radius of curvature; SD: Standard deviation; SE: Spherical equivalent}

\section{Acknowledgements}

None.

\section{Funding}

This research was supported by grants from Science and Technology Planning Project of Guangdong and Hainan Province (ZDXM2014077, 2013B090200057).

\section{Availability of data and materials}

All the data supporting the conclusions of this article are contained within the manuscript.

\section{Authors' contributions}

All authors $(\mathrm{MH}, \mathrm{HD}, \mathrm{HH}, \mathrm{CZ}, \mathrm{LL}, \mathrm{XZ})$ conceived of and designed the experimental protocol. $\mathrm{MH}$ and $\mathrm{HD}$ collected the data. All authors (MH, HD, HH, CZ, LL, XZ) were involved in the analysis. $\mathrm{MH}$ wrote the first draft of the manuscript. $\mathrm{HD}, \mathrm{HH}$, $C Z, L L$, and XZ reviewed and revised the manuscript and produced the final version. All authors read and approved the final manuscript.

\section{Competing interests}

The authors declare that they have no competing interests.

\section{Consent for publication}

Informed consents for publication were obtained from the subjects' parents before examination. All authors have seen the manuscript and approved to publish it to the journal.

\section{Ethics approval and consent to participate}

The study followed the tenets of the Declaration of Helsinki and the protocol was reviewed and approved by the Ethics Review Board of Hainan Eye Hospital of the Zhongshan Ophthalmic Center (HNEH2015-015). Informed consents for participation were obtained from the subjects' parents before examination.

\section{Publisher's Note}

Springer Nature remains neutral with regard to jurisdictional claims in published maps and institutional affiliations.

Received: 26 June 2016 Accepted: 8 May 2017

Published online: 17 May 2017

\section{References}

1. Glass DH, Roberts CJ, Litsky AS, Weber PA. A viscoelastic biomechanical model of the cornea describing the effect of viscosity and elasticity on hysteresis. Invest Ophthalmol Vis Sci. 2008;49(9):3919-26.

2. Hatami-Marbini H. Hydration dependent viscoelastic tensile behavior of cornea. Ann Biomed Eng. 2014;42(8):1740-8.

3. Lanza M, Cennamo M, laccarino S, Irregolare C, Rechichi M, Bifani M, Gironi CU. Evaluation of corneal deformation analyzed with scheimpflug based device in healthy eyes and diseased ones. Biomed Res Int. 2014;2014:748671.

4. Salvetat ML, Zeppieri M, Tosoni C, Felletti M, Grasso L, Brusini P. Corneal deformation parameters provided by the corvis-ST pachy-tonometer in healthy subjects and glaucoma patients. J Glaucoma. 2015;24(8):568-74.

5. Luce DA. Determining in vivo biomechanical properties of the cornea with an ocular response analyzer. J Cataract Refract Surg. 2005;31(1):156-62.

6. Shah A, Lascaratos G, Garway-Heath DF, Foster PJ, Barton K. Longitudinal study of iris concavity, corneal biomechanics, and correlations to ocular biometry in a cohort of 10- to 12-year-old UK schoolboys: 2-year follow-up data. Invest Ophthalmol Vis Sci. 2014;55(7):4645-50.

7. Shah A, Low S, Garway-Heath DF, Foster PJ, Barton K. Iris concavity, corneal biomechanics, and their correlations with ocular biometry in a cohort of 10- to 12-year-old UK school boys: baseline data. Invest Ophthalmol Vis Sci. 2014:55(5):3303-10.

8. Touboul D, Roberts C, Kerautret J, Garra C, Maurice-Tison S, Saubusse E, Colin J. Correlations between corneal hysteresis, intraocular pressure, and corneal central pachymetry. J Cataract Refract Surg. 2008;34(4):616-22.

9. Shah S, Laiquzzaman M, Cunliffe I, Mantry S. The use of the Reichert ocular response analyser to establish the relationship between ocular hysteresis, corneal resistance factor and central corneal thickness in normal eyes. Cont Lens Anterior Eye. 2006;29(5):257-62.

10. Huang Y, Huang C, Li L, Qiu K, Gong W, Wang Z, Wu X, Du Y, Chen B, Lam $D S$, et al. Corneal biomechanics, refractive error, and axial length in Chinese primary school children. Invest Ophthalmol Vis Sci. 2011;52(7):4923-8.

11. Bueno-Gimeno I, Gene-Sampedro A, Pinero-Llorens DP, Lanzagorta-Aresti A, Espana-Gregori E. Corneal biomechanics, retinal nerve fiber layer, and optic disc in children. Optom Vis Sci. 2014;91(12):1474-82.

12. Lanza M, Cennamo M, laccarino S, Romano V, Bifani M, Irregolare C, Lanza A. Evaluation of corneal deformation analyzed with a Scheimpflug based device. Cont Lens Anterior Eye. 2015;38(2):89-93.

13. Valbon BF, Ambrosio RJ, Fontes BM, Luz A, Roberts CJ, Alves MR. Ocular biomechanical metrics by CorVis ST in healthy Brazilian patients. J Refract Surg. 2014;30(7):468-73. 
14. Valbon BF, Ambrosio RJ, Fontes BM, Alves MR. Effects of age on corneal deformation by non-contact tonometry integrated with an ultra-high-speed (UHS) Scheimpflug camera. Arq Bras Oftalmol. 2013;76(4):229-32.

15. Hon Y, Lam AK. Corneal deformation measurement using Scheimpflug noncontact tonometry. Optom Vis Sci. 2013;90(1):e1-8.

16. Tian L, Wang D, Wu Y, Meng X, Chen B, Ge M, Huang Y: Corneal biomechanical characteristics measured by the CorVis Scheimpflug technology in eyes with primary open-angle glaucoma and normal eyes. Acta Ophthalmol. 2015. doi: 10.1111/aos.12672. [Epub ahead of print].

17. Ye C, Yu M, Lai G, Jhanji V. Variability of corneal deformation response in normal and keratoconic eyes. Optom Vis Sci. 2015;92(7):e149-53.

18. Wang $W, D u S$, Zhang $X$. Corneal deformation response in patients with primary open-angle glaucoma and in healthy subjects analyzed by corvis ST. Invest Ophthalmol Vis Sci. 2015;56(9):5557-65.

19. Bao F, Chen H, Yu Y, Yu J, Zhou S, Wang J, Wang Q, Elsheikh A. Evaluation of the shape symmetry of bilateral normal corneas in a Chinese population. PLoS One. 2013;8(8), e73412.

20. Li Y, Bao FJ. Interocular symmetry analysis of bilateral eyes. J Med Eng Technol. 2014;38(4):179-87.

21. Durr GM, Auvinet E, Ong J, Meunier J, Brunette I. Corneal shape, volume, and interocular symmetry: parameters to optimize the design of biosynthetic corneal substitutes. Invest Ophthalmol Vis Sci. 2015;56(8):4275-82.

22. Zheng X, Bao F, Geraghty B, Huang J, Yu A, Wang Q. High intercorneal symmetry in corneal biomechanical metrics. Eye Vis (Lond). 2016;3:7.

23. Kotecha A. What biomechanical properties of the cornea are relevant for the clinician? Surv Ophthalmol. 2007:52 Suppl 2:S109-14.

24. Boote $C$, Hayes $S$, Abahussin M, Meek KM. Mapping collagen organization in the human cornea: left and right eyes are structurally distinct. Invest Ophthalmol Vis Sci. 2006:47(3):901-8.

25. Bak-Nielsen S, Pedersen IB, Ivarsen A, Hjortdal J. Repeatability, reproducibility, and age dependency of dynamic Scheimpflug-based pneumotonometer and its correlation with a dynamic bidirectional pneumotonometry device. Cornea. 2015;34(1):71-7.

26. Nemeth G, Hassan Z, Csutak A, Szalai E, Berta A, Modis LJ. Repeatability of ocular biomechanical data measurements with a Scheimpflug-based noncontact device on normal corneas. J Refract Surg. 2013;29(8):558-63.

27. Chen X, Stojanovic A, Hua Y, Eidet JR, Hu D, Wang J, Utheim TP. Reliability of corneal dynamic scheimpflug analyser measurements in virgin and postPRK eyes. PLoS One. 2014;9(10), e109577.

28. Malik NS, Moss SJ, Ahmed N, Furth AJ, Wall RS, Meek KM. Ageing of the human corneal stroma: structural and biochemical changes. Biochim Biophys Acta. 1992;1138(3):222-8.

29. Daxer A, Misof K, Grabner B, Ettl A, Fratzl P. Collagen fibrils in the human corneal stroma: structure and aging. Invest Ophthalmol Vis Sci. 1998;39(3):644-8.

30. Dupps WJ, Wilson SE. Biomechanics and wound healing in the cornea. Exp Eye Res. 2006;83(4):709-20

31. Kida T, Liu JH, Weinreb RN. Effects of aging on corneal biomechanical properties and their impact on 24-h measurement of intraocular pressure. Am J Ophthalmol. 2008;146(4):567-72.

\section{Submit your next manuscript to BioMed Central and we will help you at every step:}

- We accept pre-submission inquiries

- Our selector tool helps you to find the most relevant journal

- We provide round the clock customer support

- Convenient online submission

- Thorough peer review

- Inclusion in PubMed and all major indexing services

- Maximum visibility for your research

Submit your manuscript at www.biomedcentral.com/submit

) Biomed Central 